\title{
Predisposición al cambio organizacional en los servidores públicos administrativos de las unidades de gestión educativa local, Apurímac, Perú
}

\author{
Predisposition to organizational change in administrative public servers of the local \\ educational management units, Apurímac, Peru \\ Predisposição à mudança organizacional nos servidores públicos administrativos \\ das unidades locais de gestão educacional, Apurímac, Peru
}

\author{
FELIPE RAFAEL VALLE DÍAZ ${ }^{1}$ \\ FREDDY VEGA LOAYZA
}

\begin{abstract}
RESUMEN
El objetivo general de la investigación fue interpretar las diferencias en la predisposición al cambio organizacional de los servidores públicos administrativos de las Unidades de Gestión Educativa Local ubicadas en la región Apurímac, Perú. Los elementos que forman sostener las diferencias son las fuerzas a favor del cambio y las fuerzas de resistencia al cambio. La investigación está ubicada en el enfoque mixto, del tipo básica, diseño comparativo y la muestra intencional, no probabilística. La prueba estadística aplicada fue la $t$ de muestras independientes. Los resultados nos demuestran que no existen diferencias significativas entre las Unidades de Gestión Educativa Local, en adelante UGEL; respecto a la predisposición al cambio organizacional de parte de los servidores públicos administrativos, todas presentan un mismo comportamiento de rutina fortalecida que deriva en una conformidad de acciones, una aversión al riesgo sostenido en el miedo a la adaptación tecnológica y competir, además que la estructura flexible permite una comunicación y organización informal sólida que no ayuda a impulsar el cambio organizacional.
\end{abstract}

Palabras clave: predisposición; cambio; organización; servidor público.

\begin{abstract}
The general objective of the research was to interpret the differences in the predisposition to organizational change of the administrative public servants of the Local Educational Management Units, located in Apurimac region, Peru. The elements that make up the differences are the forces in favor of change and the forces of resistance to change. The research is located in the mixed approach, basic research, whose design was comparative and the sample was intentional, non-probabilistic. The statistical test applied was the " $\mathrm{t}$ " of independent samples. The results show us that there are no significant differences between the Local Educational Management Units, hereinafter LEMU; regarding the predisposition to organizational change on the part of public administrative servants, all of them present the
\end{abstract}


same strengthened routine behavior that results in a conformity of actions, an aversion to risk sustained in fear of technological adaptation and competition. In addition to the flexible structure, it allows for robust informal communication and organization that does not help drive organizational change.

Keywords: predisposition; change; organizational; public server.

\section{RESUMO}

O objetivo geral da pesquisa foi interpretar as diferenças na predisposição à mudança organizacional dos servidores públicos administrativos das Unidades de Gestão Educacional Local, localizado na região de Apurimac, Peru. Os elementos que compõem as diferenças são as forças a favor da mudança e as forças da resistência à mudança. A pesquisa está localizada na abordagem mista, uma pesquisa básica, cujo desenho foi comparativo e a amostra foi intencional, não probabilístico. O teste estatístico aplicado foi o " $\mathrm{t}$ " de amostras independentes. Os resultados mostram que não há diferenças significativas entre as Unidades de Gestão Educacional Local, em diante UGEL; sobre a predisposição à mudança organizacional por parte dos servidores públicos, todos eles exibem o mesmo comportamento rotineiro fortalecido que resulta em uma conformidade das ações, uma aversão ao risco sustentada pelo medo de adaptação tecnológica e competição. Além da estrutura flexível, permite comunicação e organização informais robustas que não ajudam a impulsionar a mudança organizacional.

Palavras-chave: predisposição; mudança; organizacional; servidor público.

\section{INTRODUCCIÓN}

A nivel mundial, las organizaciones inmersas en el mercado global, deben tener una cultura de fortalecer la predisposición al cambio. Este reto es necesario para que desarrollen innovación de procedimientos, productos y servicios; de lo contrario tendrán serias limitaciones para competir en el mercado global. Este principio, es adoptado por una considerable cantidad de organizaciones, bajo régimen privado, donde la inversión de cada dólar, debe transformarse en superior. Además, estas organizaciones que buscan recuperar la inversión en un mediano plazo, tienden a obtener lucro y la iniciativa empresarial, se centra por obtener, mantener el margen de ganancia. Es muy diferente las instituciones u organizaciones cuyo objeto de constitución es servir para alcanzar beneficios sociales, es decir que los usuarios sean quienes reciban el servicio adecuadamente, siendo un beneficio para él. Es aquí, donde nacen los principios de atención oportuna, respuesta rápida, empatía con el usuario del servicio, y que es determinante que el servidor público que presta el servicio, tenga una formación suficiente, habilidades blandas para el trato y comunicación, y la seguridad del proceso del servicio que prestará. Esto, significa que siempre, cualquier servidor público, debe tener un sesgo hacia la adaptación tecnológica, alcanzar y justificar incentivos económicos, mejorar la comunicación, cambiar hábitos negativos, reducir conflictos, buscar una mayor cohesión grupal y salir de su zona de confort. La realidad peruana, en los sectores sociales, caso Educación, si bien el estado peruano, desembolsa e invierte una considerable 
cantidad de recursos económicos año a año; y nunca se contrae en presupuesto en estos dos sectores, existe una considerable cantidad de actividades, procesos que son ineficientes, que muchas veces son productos del desenvolvimiento individual a grupal; como también forzado por la rigidez normativa y funcional. Además, la burocracia; es la que consolida los hábitos, zona de confort, limitaciones de ejecución de función, prioridades inmediatas directas, entre otros procesos y actividades. Por ello, en este sector, se observa y evidencia por información indirecta, que existen problemas funcionales relacionados al compromiso del servidor público, que merma el rendimiento y productividad laboral, derivando en una débil imagen y posición institucional. Esto trae como consecuencia que existe insatisfacción en el usuario que toma el servicio, que busca mediante su atención un beneficio.

La investigación tuvo como problema general, el siguiente: ¿Cuáles son las diferencias en la predisposición al cambio organizacional en los servidores públicos administrativos de las Unidades de Gestión Educativa Local, región Apurímac, Perú 2019? Los elementos que forman sostener las diferencias son: Las fuerzas a favor del cambio y las fuerzas de resistencia al cambio. El objetivo general de la investigación fue: Interpretar las diferencias en la predisposición al cambio organizacional de los servidores públicos de las Unidades de Gestión Educativa Local, región Apurímac, Perú, 2019.

El propósito de la investigación es, primero, precisar las diferencias en la predisposición al cambio organizacional en el sector social educativo a nivel administrativo, eslabón primordial de estrategia y política pública del estado peruano. Segundo, los resultados permitirán conocer el estado actual de la predisposición al cambio organizacional en los servidores públicos administrativos, ubicados en el sector educativo de las distintas provincias de la región Apurímac.

Para Hernández, Fernández \& Baptista (2014). La investigación está ubicada en el enfoque mixto, por la característica de la variable de estudio. Para Carrasco Díaz (2005) es una investigación de tipo básica, que permite aportar conocimiento actual de la variable de estudio. Según Ortiz \& García (2008) el diseño es no experimental, ligado al diseño comparativo, porque describe, analiza diferencias entre las muestras. Las técnicas de investigación fueron: La entrevista, encuesta semi estructurada. Los instrumentos de recojo de datos fueron: ficha de entrevista y cuestionario. Las unidades de análisis fueron servidores públicos administrativos, cuya muestra fue intencionada. Las Unidades de Gestión Educativa Local, que se visitó y aplicó los instrumentos fueron: Abancay, Andahuaylas, Chincheros, Grau, Tambobamba. Las hipótesis estadísticas fueron:

Ho: No existe diferencias significativas si las medias son similares y p-valor $>0.05$.

Ha: Existe diferencias significativas si las medias son dispersas y p-valor $<0.05$.

\section{REVISIÓN DE LA BIBLIOGRAFÍA}

Para Barba (2000) en su estudio analizó que los ejes comunes y las características generales que se asocian al cambio en los paradigmas de las teorías administrativas entre los que se pueden apreciar: el cambio en la racionalidad gerencial, la incorporación de los aspectos simbólicos, la modificación sustantiva de las estrategias empresariales, la reflexi- 
vidad de las nuevas instituciones y el cambio en la lógica de la organización del trabajo, por mencionar algunos de los aspectos más relevantes (p. 12). Además, Barba (2000) el nuevo modelo de organización posmoderna o flexible surge en medio de los fenómenos de mundialización, globalización y regionalización; este cambio de paradigma que permite el cambio organizacional, se origina en los procesos que se desarrollan en el marco de la mundialización y que se expresan en los espacios económicos, socioculturales y políticos; pero sobre todo muy ceñido a lo expuesto por Warren Bennis, citado por Barba (2000) donde se refiere a la organización del siglo XXI en términos de la necesidad de reinventarse dada la obsolescencia de muchos postulados tradicionales (p. 16). Asimismo, Barba (2000) indica que es bajo este contexto en el que han tenido lugar la tercera revolución industrial, la consolidación del proceso de globalización y la formación de los bloques comerciales (p. 12). lo que ha generado la competitividad global, centrado en la innovación tecnológica y de productos, que para ello se requieren arreglos estructurales que promuevan una mayor autonomía de trabajo, la descentralización de responsabilidades y una alta profesionalización de sus empleados. Barba (2000) la flexibilidad de estructuras implica el reconocimiento de la importancia del crecimiento del ámbito informal para facilitar nuevas formas de cooperación, la comunicación y la movilización de saberes. La supremacía de las industrias de electrónica, química y automotriz son desplazadas aceleradamente por la microelectrónica y la computación aplicada a los procesos de producción y organizacionales. Esta revolución tecnológica se convierte en una de las condiciones sustantivas para la modernización y la flexibilización de las instituciones (p. 12). Un ejemplo de modelo emergente de organizaciones fue Japón, quien, a partir de los años 70 y 80 del siglo XX, se perfiló como uno de los principales competidores en el mercado internacional, desplazando a países industrializados, entre ellos a los Estados Unidos. Este desplazamiento fue considerado como una seria amenaza económica y el éxito se fundamentó, entre otros factores, en el modelo de organización que en la práctica mostraba ser uno de los más eficientes, cuyos postulados teóricos se habían conformado desde principios de siglo XX sobre la base de las propuestas de Frederick Taylor.

Díaz-Barrios (2005) centró en identificar aquellos valores, condición sine-qua-non (una condición que necesariamente ha de cumplirse o es indispensable para que suceda o se cumpla algo), para el logro de los objetivos de cambio y analizarlos afín de determinar sus indicadores, con el objeto de presentar una herramienta a las organizaciones que les facilite adaptarse a los nuevos tiempos. Siguiendo a Díaz-Barrios (2005) se encontró que los valores: delegación, comunicación, colaboración, participación y aprendizaje, son esenciales en los cambios integrales de esta era, tal como fue expuesto por Martínez, citado por Díaz, respecto a que los modelos clásicos de cambio, no son ahora suficientes para lograr los cambios deseados. El mundo de hoy se caracteriza por estar interconectado de forma global, que todos los fenómenos son recíprocamente interdependientes y que para describirlo necesitamos una perspectiva más amplia, holista y ecológica que no pueden ofrecernos las concepciones reduccionistas. Para Gibson (1996) demuestra que solo a partir de la reeducación se podrá hacer transformación; y no se podrá hacer ese cambio por la razón o el poder; son otros tiempos, contextos diferentes al primer lustro y décadas siguientes del siglo XX. La primera conclusión de Díaz-Barrios (2005) es que el cambio organizacional, significa un cambio en los patrones de conducta de los empleados, concentrarse en sus habilidades, percepciones, actitudes y expectativas (p. 606). 
Asimismo, Daft (1998) precisa que el nuevo paradigma organizacional posmoderno, emergente; nos indica que las cualidades: igualdad, delegación, relaciones horizontales, construcción por consenso, son importantes en la organización posmoderna, toman mayor relevancia, en respuesta a las condiciones turbulentas y caóticas del entorno ( $\mathrm{p}$.609). Es más, la organización debe tener una estructura flexible y descentralizada con bordes difusos, comunicación informal, liderazgo servil, control a través de la auto regulación y con el igualitarismo como guía principal. En este ambiente, lo único que puede salvar a una organización es un cambio hacia una fuerte cultura, fuertes valores que la guíen por encima de sus líderes hacia las necesidades del cliente, que responda a sus obligaciones a través del autocontrol y la responsabilidad propia, que potencie a los trabajadores a desarrollar habilidades competitivas a través de su aprendizaje continuo (p. 609). Blanchard \& O'Connor (1997) mencionan que el cambio organizacional, es "el éxito verdadero, no proviene de proclamar nuestros valores, sino de ponerlos en práctica consecuentemente todos los días" (p. 612). Entonces una condición de la existencia y desarrollo de una cultura organizacional predispuesta al cambio, es que el primer paso es conocer los valores requeridos, el segundo paso es saber si en la empresa están presentes o no estos valores requeridos. Si lo están, debe alinearse la organización alrededor de ellos y si no lo están, entonces habrá que desarrollarlos antes de poner en práctica cualquier otro cambio. Díaz-Barrios (2005) concluye que los cambios organizacionales tienen que girar alrededor del individuo y de los valores que pueden moverlo hacia la nueva cultura (delegación de autoridad, comunicación abierta, participación, colaboración y aprendizaje continuo). El diagnóstico organizacional a través de estos valores permitirá conocer si están sentadas las bases para comenzar el camino del cambio organizacional, o si por el contrario, tiene que, como paso previo, iniciarse un proceso de alineación integral de esos valores a fin de modificar la cultura y hacerla proclive al cambio, disminuyendo la resistencia e incrementando las posibilidades de éxito, con una reducción de costos, tiempo y esfuerzo en la consecución de los objetivos finales que se plantearon (p. 624).

López, Restrepo de Ocampo \& López Velásquez (2013), sobre el estudio de la Resistencia al cambio en organizaciones modernas, proponen una mirada del ser humano como pilar de todos los cambios al interior de la organización y el proceso de cambio; que se inicia con el cambio personal y como las organizaciones modernas son conscientes de ello. El cambio, es un proceso a través del cual se pasa de un estado a otro, generándose modificaciones o alteraciones de carácter cuantitativo y/o cualitativo de la realidad. El cambio, es un fenómeno social que ocurre en diversos contextos, con diferentes magnitudes, y con variados tipos y manifestaciones. Este ha sido y es, inherente a la sociedad y al hombre desde el mismo inicio de su existencia y aunque su ocurrencia es diaria, las personas no pueden asumir sus repercusiones tan rápido como para no sufrir trastornos por ello, tal como lo indica León, (s.f., citado por López, Restrepo de Ocampo \& López Velásquez, 2013). Asimismo, el cambio es un fenómeno conceptualmente simple en que intervienen dos conceptos bien identificados: una situación inicial de la que queremos salir y una situación objetivo que juzgamos como relativamente ventajosa. La resistencia al cambio se produce tanto en el ámbito organizacional como personal, pudiendo estar ligada a la personalidad, al sistema social y al modo de implementación de cambio; adoptando diversas formas. Robbins (2004, citado por López, Restrepo de Ocampo \& López Velásquez, 2013), señala que la resistencia puede ser abierta, cuando se manifiesta en huelgas, menor productividad, trabajo defectuoso o incluso sabotaje, o la resistencia puede ser encubierta cuando se manifiesta en demoras, 
ausentismo mayor, solicitudes de traslado, renuncias, pérdida de la motivación, moral más baja y tasas más altas de accidentes o errores (p. 150). En este sentido se expresa que una de las formas más dañinas de la resistencia es la falta de participación y de compromiso de los empleados con los cambios propuestos, incluso cuando tienen oportunidad de participar, demostrado por Hellriegel y Cols (s.f., citado por López, Restrepo de Ocampo \& López Velásquez (2013, p. 130), existen presiones, sumamente importantes, que enfrentan las empresas para el cambio. Ellas son:

1. Globalización de los mercados, enfrentar a una competencia global en una escala sin precedentes. Cada vez más los principales participantes de la economía del mundo son empresas internacionales o multinacionales. El surgimiento de estas organizaciones globales crea presiones sobre las compañías nacionales a fin de rediseñar $\mathrm{y}$, a su vez, internacionalizar las operaciones.

2. La difusión de la tecnología de la información y de las redes de computación, para enfrentarse a la competencia internacional hace falta flexibilidad, ello lo permite la tecnología de la información.

3. Los cambios en la naturaleza de la fuerza laboral empleada por las organizaciones, debe ser cada vez más educada, menos sindicalizada y se caracteriza por valores y aspiraciones cambiantes. Aunque los valores y aspiraciones cambiantes no disminuyen las aspiraciones a mayores recompensas que desean las personas del trabajo y el equilibrio que buscan entre el trabajo y otros aspectos de sus vidas.

La teoría de sostén para la investigación de López, Restrepo de Ocampo \& López Velásquez (2013) se basó en el enfoque sistémico, porque proporcionó una forma clara, directa de pensar el cambio organizacional. El modelo de sistemas del cambio, describe la organización en forma de seis variables que se afectan en forma recíproca, es decir, que son interdependientes, ellas son: la gente, la cultura, la tarea, la tecnología, el diseño y la estrategia. La variable de la gente se aplica a las personas que trabajan para la organización, incluyendo sus diferencias individuales, personalidades, actitudes, percepciones, atribuciones. La variable de la cultura refleja las creencias, valores, expectativas y normas compartidas de los miembros de la organización. La variable de la tarea incluye la naturaleza del trabajo en sí. La variable de la tecnología abarca los métodos y técnicas de solución de problemas y la aplicación de conocimiento a diversos procesos organizacionales. La variable del diseño es la estructura organizacional formal y sus sistemas de comunicación, control, autoridad y responsabilidad. La variable estrategia abarca el proceso de planeación de la organización. (p. 151). Una ventaja de este enfoque de sistemas es que ayuda a los directivos y empleados a pensar en las relaciones mutuas que se dan entre estas seis variables. López, Restrepo de Ocampo \& López Velásquez (2013) nos brinda afirmación sobre que el enfoque de sistemas afirma que no es posible cambiar parte de la organización sin cambiarla toda.

El enfoque sistémico tuvo como impulsor a Kurt Lewin, quién estructuró un proceso en la tentativa de tomar un cambio efectivo y duradero. Básicamente, la idea es de descongelar valores antiguos, cambiar y, re congelar estos nuevos valores (p. 152). Descongelar; implica tornar tan obvia la necesidad de cambio a punto del individuo, del grupo o de la organización y poder fácilmente verla y aceptarla. El cambio; implica un agente de cambio entrenando, que ira a liderar a los individuos, los grupos o toda la organización durante el 
proceso, es decir; debe alimentar hacia los nuevos valores, aptitudes y comportamientos a través de los procesos de identificación e internalización. Los miembros de la organización irán a identificarse con los valores, aptitudes y comportamientos del agente de cambio, internalizándolos así que perciben su eficacia en el desempeño (p. 152) Re congelar; significa transformar en regla general un nuevo padrón de comportamiento, usando para esto mecanismos de apoyo o refuerzo, de modo que se torne una nueva norma. El cuarto concepto, es el de la transición. Para López, Restrepo de Ocampo \& López Velásquez (2013) la transición es esa especie de situación intermedia donde notamos las trabas, las dificultades y los costes del cambio y donde, desafortunadamente, no hemos aún abandonado completamente las desventajas originales ni hemos obtenido todavía los beneficios que esperamos. Es el momento en que el cambio es más frágil (p. 153). El desafío es romper el equilibrio existente, en todo este proceso es claro: consiste en minimizar el decaimiento temporal, pero sin resignar la profundidad que el cambio requiere y, por otra parte, en reducir la duración de la transición, pero atendiendo a la capacidad de la organización y de los individuos para absorber los nuevos conceptos y adquirir las nuevas capacidades que se requieran para asegurar los resultados finales y su estabilidad en el tiempo.

Acosta (2002), con respecto a las organizaciones empresariales, se centra en las dimensiones: las estructuras, la tecnología, el comportamiento humano y la cultura; donde se localizan los cambios, explora lo que algunos autores dicen sobre el cambio, revisa varias propuestas sobre las razones que llevan al cambio en las organizaciones, expone las modalidades en que cambian las organizaciones y, finalmente, como inferencia de lo expuesto, concluye en un concepto de cambio organizacional. Acosta (2002) las organizaciones se ven compelidas a cambiar por motivos relacionados con su propia sobrevivencia, su evolución y como respuesta a la búsqueda de un crecimiento programado. Se puede decir que las organizaciones cambian para responder a las exigencias internas que tocan con su propia evolución y entropía, tanto como para enfrentar las exigencias externas relacionadas con su propio crecimiento y con el mundo de la competencia en que se inscriben, pero este cambio se logra siempre y cuando los agentes internos contribuyan a consolidar este objetivo de cambio, de lo contrario las fuerzas en contra, inmersas en la resistencia al cambio mermarán los objetivos de mejora. Acosta (2002) señalan que las empresas buscan el cambio porque de esa manera logran avances frente a la competencia, lo cual significa que así esperan:

1. encontrar sus propias maneras de administrarse, de potenciarse y de afrontar los retos del medio,

2. mejorar su capacidad para aprovechar las oportunidades que les ofrece el mercado y

3. lograr claridad en un medio donde reina la incertidumbre.

Acosta (2002) nos precisan que las fuerzas obstaculizadoras al cambio, se ubican en aquellas personas que rechazan "las razones" por las cuales la organización debe cambiar. Tal rechazo genera una actitud cuyo componente racional queda explícito que el funcionario lo considera perjudicial para la organización, apoya la aparición de un componente afectivo negativo al individuo y estimula la aparición del componente conductual de actuación en contra del cambio. Adicionalmente, la persona puede considerarlo favorable o desfavorable a sus intereses personales y actuar en consecuencia. Sin embargo, ambos nos aclaran que, la resistencia al cambio, según su forma de expresión puede ser de dos tipos: Tipo A, es abier- 
ta e inmediata, la cual ocurre cuando los trabajadores responden con trabajo lento, quejas y amenazas manifiestas. Tipo B, es implícita y diferida cuando se trata de una reacción mínima al momento del cambio; genera resistencia en el largo plazo, acumula resentimiento que explota después sin conexión explícita con el evento de cambio, produce pérdida de lealtad, desmotivación, ausentismo, baja en la eficiencia y en la eficacia. Ambos escenarios, deben ser identificados, analizados e iniciar un plan de sensibilización y recuperación del talento humano que presenta resistencia al cambio. Se puede concluir, en general, que los cambios se producen en las áreas funcionales, en las unidades de negocios en algunos procesos específicos como también se producen en la totalidad de la organización. Y no es que sean muy distintos estos cambios, sino que cada propuesta ve la organización desde un enfoque diferente. Por eso se puede comenzar diciendo que el cambio organizacional ocurre en la totalidad de la organización o en sus estructuras, en los procesos, las áreas o las dimensiones (política, de funcionamiento interno, de relaciones exteriores, entre otros).

La Teoría de soporte para la investigación: Predisposición al cambio..., parte del modelo de Kurt Lewin (s.f., citado en López, Restrepo de Ocampo \& López Velásquez, 2013) y en lo señalado por Jones (2008) se demostró que el comportamiento, rendimiento y posición de una organización es el resultado de las fuerzas a favor del cambio y de las fuerzas que se resisten al cambio, el equilibrio entre estas dos fuerzas genera el statu quo, donde las compensaciones de las fuerzas, establecen parámetros de comportamiento que se conservan, llamado el equilibrio cuasi estacionario, para alterar el equilibrio cuasi se debe incidir en implementar, fortalecer las fuerzas a favor del cambio o aprender de la oposición, reduciendo las fuerzas resistentes al cambio, para estos dos casos, siempre se debe pasar por tres fases: el descongelamiento, que significa reducir las fuerzas que conservan a la organización en su nivel real y actual de comportamiento. El cambio o movimiento, que significa pasar a un estado distinto, desde los patrones de comportamiento identificados en el descongelamiento hacia crear nuevos valores, conductas, normas, es decir el inicio de la adaptación hacia el proceso de cambio. El recongelamiento, significa que el cambio se logró, porque se consolidaron valores, conductas, normas nuevas, aceptadas, legitimadas, apoyados en factores culturales, políticos, ambientales. El segundo soporte cuyo modelo está relacionado y parte desde el modelo de Kurt Lewin, es el modelo de Burke-Litwin, el cual es una variante del modelo Kurt Lewin, donde el cambio se implementa en el desempeño individual y el organizacional. El primer cambio o primer orden es el cambio transaccional, que significa la adaptabilidad, que modifica al individuo y organización en forma evolutiva, gradual, pero mantiene su esencia. El segundo cambio o segundo orden, es el cambio transformacional, que significa la alteración, reingeniería completa de toda la organización. Estos dos momentos, según Lewin, Burke y Litwin, existen fuerzas a favor y resistentes al cambio, cuya interacción permite que la organización pueda adaptarse y vivir a las transformaciones de su entorno. Jones (2008) refiere que las fuerzas a favor del cambio, son aquellas fuerzas que a partir de su implementación generan un impacto en la organización, independiente de su ubicación sea interna o externa, porque contribuyen al cambio y este fortalece la competitividad, gracias a la adaptación y la trasformación del individuo y organización, es decir mejora, impulsa el desarrollo y cultura organizacional. Las fuerzas resistentes al cambio, son aquellas que insisten en mantener el statu quo del individuo y la organización, frenan la adaptación e impiden la transformación, por intereses, posición, individuales, grupales al interior de la organización, presentan aversión al riesgo, no piensan salir de la zona de confort, es todo lo contrario para el desarrollo y cultura organizacional. 


\section{RESULTADOS}

\section{Resultado del Alfa de Cronbach}

El Alfa de Cronbach para el instrumento aplicado en los servidores públicos administrativos, ubicados en las Unidades de Gestión Educativa Local, obtuvo un promedio 0.87, el cual presenta una alta fiabilidad.

\section{Resultado de la estadística inferencial: prueba $t$}

Tabla 1.

Prueba t para muestras independientes

\begin{tabular}{cccccccc}
\hline \hline & & & \multicolumn{2}{c}{$\begin{array}{c}\text { Prueba de Levene de } \\
\text { igualdad de varianzas }\end{array}$} & & \multicolumn{3}{c}{ prueba t para la igualdad de medias } \\
\cline { 2 - 7 } UGEL Abancay y Grau & $\mathrm{F}$ & Sig. & $\mathrm{t}$ & $\mathrm{gl}$ & $\begin{array}{c}\text { Sig. } \\
\text { (bilateral) }\end{array}$ & $\begin{array}{c}\text { Diferencia de } \\
\text { medias }\end{array}$ \\
\cline { 2 - 7 } FAVOR & $\begin{array}{c}\text { Se asumen va- } \\
\text { rianzas iguales }\end{array}$ & .124 & .727 & -.115 & 35 & .909 & -.01765 \\
\cline { 2 - 7 } RESISTENCIA & $\begin{array}{c}\text { No se asumen } \\
\text { varianzas iguales }\end{array}$ & & & -.114 & 32.976 & .910 & -.01765 \\
\hline $\begin{array}{c}\text { Se asumen va- } \\
\text { rianzas iguales }\end{array}$ & $\begin{array}{c}\text { No se asumen } \\
\text { varianzas iguales }\end{array}$ & .041 & .840 & -.411 & 35 & .684 & -.06765 \\
\hline \hline
\end{tabular}

Fuente: UGEL región Apurímac. 2019. Elaboración con soporte SPSS 23

Dados los resultados a partir de los datos obtenidos en las Unidades de Gestión Educativa Local de Abancay y Grau, podemos confirmar que no existen diferencias significativas hacia una predisposición al cambio organizacional. Los resultados indican que el p-valor es mayor a 0.05 , lo cual determina que se rechaza la hipótesis alterna y se aprueba la hipótesis nula; es decir significa que no existe diferencias significativas a favor del cambio y no existe diferencias significativas en la resistencia al cambio en las UGEL de la región Apurímac. Los resultados nos demuestran que, los comportamientos de los servidores públicos administrativos a favor del cambio y la resistencia al cambio son parecidos, similares en las dos UGEL comparadas.

Tabla 2.

Prueba t para muestras independientes

\begin{tabular}{clccccccc}
\hline \multirow{2}{*}{ UGEL Abancay y Andahuaylas } & \multicolumn{3}{c}{$\begin{array}{c}\text { Prueba de Levene de } \\
\text { igualdad de varianzas }\end{array}$} & \multicolumn{3}{c}{ prueba t para la igualdad de medias } \\
\cline { 2 - 8 } FAVOR & $\mathrm{F}$ & Sig. & $\mathrm{t}$ & $\mathrm{gl}$ & $\begin{array}{c}\text { Sig. (bi- } \\
\text { lateral) }\end{array}$ & $\begin{array}{c}\text { Diferencia } \\
\text { de medias }\end{array}$ \\
\hline \multirow{2}{*}{ RESISTENCIA } & $\begin{array}{l}\text { Se asumen va- } \\
\text { rianzas iguales }\end{array}$ & .305 & .585 & -.696 & 32 & .491 & -.11765 \\
\cline { 2 - 8 } & $\begin{array}{l}\text { No se asumen } \\
\text { varianzas iguales }\end{array}$ & $\begin{array}{l}\text { Se asumen va- } \\
\text { rianzas iguales }\end{array}$ & .592 & .447 & 0.000 & 32 & 1.000 & 0.00000 \\
\hline & $\begin{array}{l}\text { No se asumen } \\
\text { varianzas iguales }\end{array}$ & & & 0.000 & 30.650 & 1.000 & 0.00000 \\
\hline \hline
\end{tabular}

Fuente: UGEL región Apurímac. 2019. Elaboración con soporte SPSS 23 
Dados los resultados a partir de los datos obtenidos en las Unidades de Gestión Educativa Local de Abancay y Andahuaylas, podemos confirmar que no existen diferencias significativas hacia una predisposición al cambio organizacional. Los resultados indican que el p-valor es mayor a 0.05 , lo cual determina que se rechaza la hipótesis alterna y se aprueba la hipótesis nula; es decir significa que no existe diferencias significativas a favor del cambio y no existe diferencias significativas en la resistencia al cambio, en las UGEL de la región Apurímac. Los resultados nos demuestran que, los comportamientos de los servidores públicos administrativos a favor del cambio y la resistencia al cambio son parecidos, similares en las dos UGEL comparadas.

Tabla 3.

Prueba t para muestras independientes

\begin{tabular}{|c|c|c|c|c|c|c|c|}
\hline \multirow{2}{*}{\multicolumn{2}{|c|}{ UGEL Abancay y Tambobamba }} & \multicolumn{2}{|c|}{$\begin{array}{l}\text { Prueba de Levene de } \\
\text { igualdad de varianzas }\end{array}$} & \multicolumn{4}{|c|}{ prueba t para la igualdad de medias } \\
\hline & & $\mathrm{F}$ & Sig. & $\mathrm{t}$ & gl & $\begin{array}{l}\text { Sig. (bi- } \\
\text { lateral) }\end{array}$ & $\begin{array}{l}\text { Diferencia } \\
\text { de medias }\end{array}$ \\
\hline \multirow{2}{*}{ FAVOR } & $\begin{array}{l}\text { Se asumen va- } \\
\text { rianzas iguales }\end{array}$ & .101 & .752 & -1.929 & 33 & .062 & -.28431 \\
\hline & $\begin{array}{c}\text { No se asumen } \\
\text { varianzas iguales }\end{array}$ & & & -1.916 & 30.471 & .065 & -.28431 \\
\hline \multirow{2}{*}{ RESISTENCIA } & $\begin{array}{l}\text { Se asumen va- } \\
\text { rianzas iguales }\end{array}$ & 2.973 & .094 & -.832 & 33 & .411 & -.11765 \\
\hline & $\begin{array}{c}\text { No se asumen } \\
\text { varianzas iguales }\end{array}$ & & & -.824 & 28.663 & .417 & -.11765 \\
\hline
\end{tabular}

Fuente: UGEL región Apurímac. 2019. Elaboración con soporte SPSS 23

Dados los resultados a partir de los datos obtenidos en las Unidades de Gestión Educativa Local de Abancay y Tambobamba, podemos confirmar que no existen diferencias significativas hacia una predisposición al cambio organizacional. Los resultados indican que el p-valor es mayor a 0.05 , lo cual determina que se rechaza la hipótesis alterna y se aprueba la hipótesis nula; es decir significa que no existe diferencias significativas a favor del cambio y no existe diferencias significativas en la resistencia al cambio, en las UGEL de la región Apurímac. Los resultados nos demuestran que, los comportamientos de los servidores públicos administrativos a favor del cambio y resistencia al cambio son parecidos, similares en las dos UGEL comparadas.

Tabla 4.

Prueba t para muestras independientes

\begin{tabular}{|c|c|c|c|c|c|c|c|}
\hline \multirow{2}{*}{\multicolumn{2}{|c|}{ UGEL Abancay y Chincheros }} & \multicolumn{2}{|c|}{$\begin{array}{l}\text { Prueba de Levene de } \\
\text { igualdad de varianzas }\end{array}$} & \multicolumn{4}{|c|}{ prueba t para la igualdad de medias } \\
\hline & & $\mathrm{F}$ & Sig. & $\mathrm{t}$ & gl & $\begin{array}{l}\text { Sig. (bi- } \\
\text { lateral) }\end{array}$ & $\begin{array}{r}\text { Diferencia } \\
\text { de medias }\end{array}$ \\
\hline \multirow{2}{*}{ FAVOR } & $\begin{array}{l}\text { Se asumen va- } \\
\text { rianzas iguales }\end{array}$ & 3.846 & .059 & -1.342 & 32 & .189 & -.17647 \\
\hline & $\begin{array}{c}\text { No se asumen } \\
\text { varianzas iguales }\end{array}$ & & & -1.342 & 23.529 & .193 & -.17647 \\
\hline \multirow{2}{*}{ RESISTENCIA } & $\begin{array}{l}\text { Se asumen va- } \\
\text { rianzas iguales }\end{array}$ & 3.846 & .059 & -1.342 & 32 & .189 & -.17647 \\
\hline & $\begin{array}{c}\text { No se asumen } \\
\text { varianzas iguales }\end{array}$ & & & -1.342 & 23.529 & .193 & -.17647 \\
\hline
\end{tabular}

Fuente: UGEL región Apurímac. 2019. Elaboración con soporte SPSS 23 
Dados los resultados a partir de los datos obtenidos en las Unidades de Gestión Educativa Local de Abancay y Chincheros, podemos confirmar que no existen diferencias significativas hacia una predisposición al cambio organizacional. Los resultados indican que el p-valor es mayor a 0.05 , (a pesar que es mínima) lo cual determina que se rechaza la hipótesis alterna y se aprueba la hipótesis nula; es decir significa que no existe diferencias significativas a favor del cambio y no existe diferencias significativas en la resistencia al cambio, en las UGEL de la región Apurímac. Los resultados nos demuestran que, los comportamientos de los servidores públicos administrativos a favor del cambio y la resistencia al cambio son parecidos, similares en las dos UGEL comparadas.

Tabla 5.

Prueba t para muestras independientes

\begin{tabular}{|c|c|c|c|c|c|c|c|}
\hline \multirow{2}{*}{\multicolumn{2}{|c|}{ UGEL Grau y Chincheros }} & \multicolumn{2}{|c|}{$\begin{array}{l}\text { Prueba de Levene de } \\
\text { igualdad de varianzas }\end{array}$} & \multicolumn{4}{|c|}{ prueba t para la igualdad de medias } \\
\hline & & $\mathrm{F}$ & Sig. & $\mathrm{t}$ & $\mathrm{gl}$ & $\begin{array}{l}\text { Sig. (bila- } \\
\text { teral) }\end{array}$ & $\begin{array}{l}\text { Diferencia } \\
\text { de medias }\end{array}$ \\
\hline \multirow{2}{*}{ FAVOR } & $\begin{array}{l}\text { Se asumen va- } \\
\text { rianzas iguales }\end{array}$ & 2.654 & .112 & -1.308 & 35 & .199 & -.15882 \\
\hline & $\begin{array}{c}\text { No se asumen } \\
\text { varianzas iguales }\end{array}$ & & & -1.369 & 30.139 & .181 & -.15882 \\
\hline \multirow{2}{*}{ RESISTENCIA } & $\begin{array}{l}\text { Se asumen va- } \\
\text { rianzas iguales }\end{array}$ & 2.407 & .130 & -.804 & 35 & .427 & -.10882 \\
\hline & $\begin{array}{c}\text { No se asumen } \\
\text { varianzas iguales }\end{array}$ & & & -.848 & 28.082 & .404 & -.10882 \\
\hline
\end{tabular}

Fuente: UGEL región Apurímac. 2019. Elaboración con soporte SPSS 23

Dados los resultados a partir de los datos obtenidos en las Unidades de Gestión Educativa Local de Grau y Chincheros, podemos confirmar que no existen diferencias significativas hacia una predisposición al cambio organizacional. Los resultados indican que el p-valor es mayor a 0.05 , lo cual determina que se rechaza la hipótesis alterna y se aprueba la hipótesis nula; es decir significa que no existe diferencias significativas a favor del cambio y no existe diferencias significativas en la resistencia al cambio, en las UGEL de la región Apurímac. Los resultados nos demuestran que, los comportamientos de los servidores públicos administrativos a favor del cambio y la resistencia al cambio son parecidos, similares en las dos UGEL comparadas.

Tabla 6.

Prueba t para muestras independientes

\begin{tabular}{|c|c|c|c|c|c|c|c|}
\hline \multirow{2}{*}{\multicolumn{2}{|c|}{ UGEL Grau y Tambobamba }} & \multicolumn{2}{|c|}{$\begin{array}{l}\text { Prueba de Levene de } \\
\text { igualdad de varianzas }\end{array}$} & \multicolumn{4}{|c|}{ prueba t para la igualdad de medias } \\
\hline & & $\mathrm{F}$ & Sig. & $\mathrm{t}$ & gl & $\begin{array}{l}\text { Sig. (bi- } \\
\text { lateral) }\end{array}$ & $\begin{array}{l}\text { Diferencia } \\
\text { de medias }\end{array}$ \\
\hline \multirow{2}{*}{ FAVOR } & $\begin{array}{l}\text { Se asumen va- } \\
\text { rianzas iguales }\end{array}$ & .006 & .939 & -1.962 & 36 & .058 & -.26667 \\
\hline & $\begin{array}{c}\text { No se asumen } \\
\text { varianzas iguales }\end{array}$ & & & -1.978 & 35.926 & .056 & -.26667 \\
\hline \multirow{2}{*}{ RESISTENCIA } & $\begin{array}{l}\text { Se asumen va- } \\
\text { rianzas iguales }\end{array}$ & 2.021 & .164 & -.350 & 36 & .728 & -.05000 \\
\hline & $\begin{array}{c}\text { No se asumen } \\
\text { varianzas iguales }\end{array}$ & & & -.357 & 33.441 & .723 & -.05000 \\
\hline
\end{tabular}

Fuente: UGEL región Apurímac. 2019. Elaboración con soporte SPSS 23 
Dados los resultados a partir de los datos obtenidos en las Unidades de Gestión Educativa Local de Grau y Tambobamba, podemos confirmar que no existen diferencias significativas hacia una predisposición al cambio organizacional. Los resultados indican que el p-valor es mayor a 0.05 , lo cual determina que se rechaza la hipótesis alterna y se aprueba la hipótesis nula; es decir significa que no existe diferencias significativas a favor del cambio y no existe diferencias significativas en la resistencia al cambio, en las UGEL de la región Apurímac. Los resultados nos demuestran que, los comportamientos de los servidores públicos administrativos a favor del cambio y la resistencia al cambio son parecidos, similares en las dos UGEL comparadas.

Tabla 7.

Prueba t para muestras independientes

\begin{tabular}{|c|c|c|c|c|c|c|c|}
\hline \multirow{2}{*}{\multicolumn{2}{|c|}{ UGEL Grau y Andahuaylas }} & \multicolumn{2}{|c|}{$\begin{array}{l}\text { Prueba de Levene de } \\
\text { igualdad de varianzas }\end{array}$} & \multicolumn{4}{|c|}{ prueba t para la igualdad de medias } \\
\hline & & $\mathrm{F}$ & Sig. & $\mathrm{t}$ & $\mathrm{gl}$ & $\begin{array}{l}\text { Sig. (bi- } \\
\text { lateral) }\end{array}$ & $\begin{array}{l}\text { Diferencia } \\
\text { de medias }\end{array}$ \\
\hline \multirow{2}{*}{ FAVOR } & $\begin{array}{l}\text { Se asumen va- } \\
\text { rianzas iguales }\end{array}$ & .072 & .790 & -.642 & 35 & .525 & -.10000 \\
\hline & $\begin{array}{c}\text { No se asumen } \\
\text { varianzas iguales }\end{array}$ & & & -.636 & 32.502 & .529 & -.10000 \\
\hline \multirow{2}{*}{ RESISTENCIA } & $\begin{array}{l}\text { Se asumen va- } \\
\text { rianzas iguales }\end{array}$ & .886 & .353 & .371 & 35 & .713 & .06765 \\
\hline & $\begin{array}{c}\text { No se asumen } \\
\text { varianzas iguales }\end{array}$ & & & .366 & 31.645 & .717 & .06765 \\
\hline
\end{tabular}

Fuente: UGEL región Apurímac. 2019. Elaboración con soporte SPSS 23

Dados los resultados a partir de los datos obtenidos en las Unidades de Gestión Educativa Local de Grau y Andahuaylas, podemos confirmar que no existen diferencias significativas hacia una predisposición al cambio organizacional. Los resultados indican que el p-valor es mayor a 0.05 , lo cual determina que se rechaza la hipótesis alterna y se aprueba la hipótesis nula; es decir significa que no existe diferencias significativas a favor del cambio y no existe diferencias significativas en la resistencia al cambio, en las UGEL de la región Apuríma. Los resultados nos demuestran que, los comportamientos de los servidores públicos administrativos a favor del cambio y la resistencia al cambio son parecidos, similares en las dos UGEL comparadas.

Tabla 8 .

Prueba t para muestras independientes

\begin{tabular}{|c|c|c|c|c|c|c|c|}
\hline \multirow{2}{*}{\multicolumn{2}{|c|}{ UGEL Andahuaylas y Tambobamba }} & \multicolumn{2}{|c|}{$\begin{array}{l}\text { Prueba de Levene de } \\
\text { igualdad de varianzas }\end{array}$} & \multicolumn{4}{|c|}{ prueba t para la igualdad de medias } \\
\hline & & $\mathrm{F}$ & Sig. & $\mathrm{t}$ & $\mathrm{gl}$ & $\begin{array}{l}\text { Sig. (bi- } \\
\text { lateral) }\end{array}$ & $\begin{array}{l}\text { Diferencia } \\
\text { de medias }\end{array}$ \\
\hline \multirow{2}{*}{ FAVOR } & $\begin{array}{l}\text { Se asumen va- } \\
\text { rianzas iguales }\end{array}$ & .125 & .726 & -1.110 & 33 & .275 & -.16667 \\
\hline & $\begin{array}{c}\text { No se asumen } \\
\text { varianzas iguales }\end{array}$ & & & -1.102 & 30.001 & .279 & -.16667 \\
\hline \multirow{2}{*}{ RESISTENCIA } & $\begin{array}{l}\text { Se asumen va- } \\
\text { rianzas iguales }\end{array}$ & 5.786 & .022 & -.717 & 33 & .478 & -.11765 \\
\hline & $\begin{array}{c}\text { No se asumen } \\
\text { varianzas iguales }\end{array}$ & & & -.706 & 25.140 & .486 & -.11765 \\
\hline
\end{tabular}

Fuente: UGEL región Apurímac. 2019. Elaboración con soporte SPSS 23 
Dados los resultados a partir de los datos obtenidos en las Unidades de Gestión Educativa Local de Andahuaylas y Tambobamba, podemos confirmar que no existen diferencias significativas hacia una predisposición al cambio organizacional. Los resultados indican que el p-valor es mayor a 0.05 , lo cual determina que se rechaza la hipótesis alterna y se aprueba la hipótesis nula; es decir significa que no existe diferencias significativas a favor del cambio; sin embargo, es el primer caso donde si existe diferencias significativas en la resistencia al cambio, en las UGEL de la región Apurímac. Los resultados nos demuestran que, los comportamientos de los servidores públicos administrativos a favor del cambio son parecidos, similares, lo contrario es en la resistencia al cambio, donde no son parecidos, no son similares, en las dos UGEL comparadas.

Tabla 9.

Prueba t para muestras independientes

\begin{tabular}{llccccccc}
\hline \hline \multirow{2}{*}{ UGEL Andahuaylas y Chincheros } & \multicolumn{2}{c}{$\begin{array}{c}\text { Prueba de Levene de } \\
\text { igualdad de varianzas }\end{array}$} & \multicolumn{3}{c}{ prueba t para la igualdad de medias } \\
\cline { 2 - 8 } FAVOR & $\mathrm{F}$ & Sig. & $\mathrm{t}$ & $\mathrm{gl}$ & $\begin{array}{c}\text { Sig. (bi- } \\
\text { lateral) }\end{array}$ & $\begin{array}{c}\text { Diferencia } \\
\text { de medias }\end{array}$ \\
\hline \multirow{3}{*}{ RESISTENCIA } & $\begin{array}{l}\text { Se asumen va- } \\
\text { rianzas iguales }\end{array}$ & 1.113 & .299 & -.436 & 32 & .665 & -.05882 \\
\cline { 2 - 8 } & $\begin{array}{l}\text { No se asumen } \\
\text { varianzas iguales }\end{array}$ & & & -.436 & 23.134 & .667 & -.05882 \\
\hline & $\begin{array}{l}\text { Se asumen va- } \\
\text { rianzas iguales }\end{array}$ & 7.071 & .012 & -1.124 & 32 & .269 & -.17647 \\
\hline & $\begin{array}{l}\text { No se asumen } \\
\text { varianzas iguales }\end{array}$ & & & -1.124 & 21.089 & .274 & -.17647 \\
\hline \hline
\end{tabular}

Fuente: UGEL región Apurímac. 2019. Elaboración con soporte SPSS 23

Dados los resultados a partir de los datos obtenidos en las Unidades de Gestión Educativa Local de Andahuaylas y Chincheros, podemos confirmar que no existen diferencias significativas hacia una predisposición al cambio organizacional. Los resultados indican que el p-valor es mayor a 0.05 , lo cual determina que se rechaza la hipótesis alterna y se aprueba la hipótesis nula; es decir significa que no existe diferencias significativas a favor del cambio; sin embargo, es el segundo caso donde si existe diferencias significativas en la resistencia al cambio, en las UGEL de la región Apurímac. Los resultados nos demuestran que, los comportamientos de los servidores públicos administrativos a favor del cambio son parecidos, similares, lo contrario es en la resistencia al cambio, donde no son parecidos, no son similares, en las dos UGEL comparadas.

Tabla 10.

Prueba t para muestras independientes

\begin{tabular}{|c|c|c|c|c|c|c|c|}
\hline \multirow{2}{*}{\multicolumn{2}{|c|}{ UGEL Tambobamba y Chincheros }} & \multicolumn{2}{|c|}{$\begin{array}{l}\text { Prueba de Levene de } \\
\text { igualdad de varianzas }\end{array}$} & \multicolumn{4}{|c|}{ prueba t para la igualdad de medias } \\
\hline & & $\mathrm{F}$ & Sig. & $\mathrm{t}$ & $\mathrm{gl}$ & $\begin{array}{l}\text { Sig. (bi- } \\
\text { lateral) }\end{array}$ & $\begin{array}{l}\text { Diferencia } \\
\text { de medias }\end{array}$ \\
\hline \multirow{2}{*}{ FAVOR } & $\begin{array}{l}\text { Se asumen va- } \\
\text { rianzas iguales }\end{array}$ & 4.367 & .044 & .987 & 33 & .331 & .10784 \\
\hline & $\begin{array}{c}\text { No se asumen } \\
\text { varianzas iguales }\end{array}$ & & & 1.000 & 28.935 & .326 & .10784 \\
\hline \multirow{2}{*}{ RESISTENCIA } & $\begin{array}{l}\text { Se asumen va- } \\
\text { rianzas iguales }\end{array}$ & .000 & .997 & -.583 & 33 & .564 & -.05882 \\
\hline & $\begin{array}{c}\text { No se asumen } \\
\text { varianzas iguales }\end{array}$ & & & -.588 & 30.640 & .561 & -.05882 \\
\hline
\end{tabular}

Fuente: UGEL región Apurímac. 2019. Elaboración con soporte SPSS 23 
Dados los resultados a partir de los datos obtenidos en las Unidades de Gestión Educativa Local de Tambobamba y Chincheros, podemos confirmar que no existen diferencias significativas hacia una predisposición al cambio organizacional. Los resultados indican que el p-valor es mayor a 0.05 , lo cual determina que se rechaza la hipótesis alterna y se aprueba la hipótesis nula; es decir significa que si existe diferencias significativas a favor del cambio; siendo el primer caso, sin embargo, no existe diferencias significativas en la resistencia al cambio, en las UGEL de la región Apurímac. Los resultados nos demuestran que, los comportamientos de los servidores públicos administrativos a favor del cambio no son parecidos, similares, lo contrario es en la resistencia al cambio, donde si son parecidos, similares, en las dos UGEL comparadas.

\section{Resultados prueba Kruskal Wallis}

Tabla 11.

Resultados prueba Kruskal Wallis

\begin{tabular}{rlll}
\hline \hline \multicolumn{3}{c}{ Rangos prueba Kruskal Wallis } \\
\hline \multirow{5}{*}{ UGEL } & $\mathrm{N}$ & $\begin{array}{c}\text { Rango } \\
\text { promedio }\end{array}$ \\
\hline \multirow{5}{*}{ FAVOR } & Abancay & 17 & 40.24 \\
\cline { 2 - 4 } & Grau & 20 & 40.95 \\
\cline { 2 - 4 } & Andahuaylas & 17 & 45.00 \\
\cline { 2 - 4 } & Tambobamba & 18 & 51.75 \\
\cline { 2 - 4 } & Chicheros & 17 & 47.38 \\
\cline { 2 - 4 } & Total & 89 & \\
\hline \multirow{5}{*}{ RESISTENCIA } & Abancay & 17 & 42.06 \\
\cline { 2 - 4 } & Grau & 20 & 44.75 \\
\cline { 2 - 4 } & Andahuaylas & 17 & 41.94 \\
\cline { 2 - 4 } & Tambobamba & 18 & 46.89 \\
\cline { 2 - 4 } & Chicheros & 17 & 49.29 \\
\cline { 2 - 4 } & Total & 89 & \\
\hline \hline
\end{tabular}

Fuente: UGEL región Apurímac. 2019. Elaboración con soporte SPSS 23

Tabla 12.

Estadísticos de prueba ${ }^{a, b}$

\begin{tabular}{lcc}
\hline & FAVOR & RESISTENCIA \\
\hline Chi-cuadrado & 5.468 & 2.095 \\
\hline Gl & 4 & 4 \\
\hline Sig. asintótica & .243 & .718 \\
\hline
\end{tabular}

a. Prueba de Kruskal Wallis

b. Variable de agrupación: UGEL

Fuente: UGEL región Apurímac. 2019. Elaboración con soporte SPSS 23 
Para la mayoría de las pruebas efectuadas, en 07 tablas de comparación, las hipótesis alternas son rechazadas y se aceptan las hipótesis nulas, es decir; significa que a nivel de la región Apurímac, en las Unidades de Gestión Educativa Local, no existen diferencias significativas a favor del cambio y en la resistencia al cambio organizacional; todos los servidores públicos administrativos ubicados en las UGEL: Abancay. Andahuaylas, Grau, presentan comportamientos parecidos, similares, entre ellas, y en 03 tablas de comparación, las hipótesis nulas o alternas, se rechazan indistintamente; los servidores públicos administrativos ubicados en las UGEL: Tambobamba y Chimcheros, presentan comportamiento en algo diferenciados, no siendo diferentes absolutos y extremos.

A partir de someter los datos a la prueba de kruskal wallis, que demuestra que tienen o provienen desde un mismo tipo de población y que confirma los resultados de la prueba $t$ de muestras independientes, respecto a la predisposición al cambio organizacional de parte de los servidores públicos administrativos, todos presentan un comportamiento de conformidad, confort, aversión al riesgo, porque si hubiera arrojado diferencias en la mayoría y no en casos aislados, podríamos haber confirmado que existe una predisposición al cambio organizacional. El valor p-valor es superior a 0.05 , para la prueba kruskal wallis, lo cual indica que las medias son iguales o muy próximas de las muestras de cada UGEL comparadas, teniendo un total de 10 pares de comparaciones sometidos a la prueba $t$ de muestras independientes y comparando las UGEL, vuelve a confirmar el resultado, que los servidores públicos administrativos de las UGEL, no presentan una predisposición hacia impulsar y lograr un cambio organizacional.

\section{DISCUSIÓN}

Según Barba (2000) analizó que los ejes comunes y las características generales que se asocian al cambio en los paradigmas de las teorías administrativas son: el cambio en la racionalidad gerencial, la incorporación de los aspectos simbólicos, la modificación sustantiva de las estrategias empresariales, la reflexividad de las nuevas instituciones y el cambio en la lógica de la organización del trabajo, se origina en los procesos que se desarrollan en el marco de la mundialización y que se expresan en los espacios económicos, socioculturales y políticos en los empleados, donde ellos inician, consolidan el cambio, además de apoyarse en la flexibilidad de los arreglos estructurales que promuevan una mayor autonomía de trabajo, la descentralización de responsabilidades y una alta profesionalización de sus empleados, sujetos a reconocer la importancia del crecimiento del ámbito informal para facilitar nuevas formas de cooperación, la comunicación y la movilización de saberes. Sin embargo, los empleados administrativos de las Unidades de Gestión Educativas Locales, presentan un espacio sociocultural de conformidad, confort respecto a la rutina, seguridad de cada proceso conocido; el espacio político se sujeta al interés por mantenerse sin competir, rechazando el riesgo. Existe una flexibilidad en los arreglos estructurales, donde la informalidad de acuerdos, tratos es fuerte; pero ello no garantiza que se pueda revertir, la indiferencia por cambiar hábitos, predisponer acciones voluntarias de mejora, restringir la movilidad de saberes; porque todos los servidores administrativos se ciñen a no arriesgar, mantener las mismas condiciones estructurales con algunos arreglos institucionales.

Para Díaz-Barrios (2005) existen condiciones que necesariamente ha de cumplirse o es 
indispensable para que suceda o se cumpla algo, para el logro de los objetivos de cambio y analizarlos afín de determinar sus indicadores, con el objeto de presentar una herramienta a las organizaciones que les facilite adaptarse a los nuevos tiempos. Los valores: delegación, comunicación, colaboración, participación y aprendizaje, son esenciales en los cambios integrales desde una perspectiva más amplia, holista y ecológica que no pueden ofrecernos las concepciones reduccionistas y solo se puede alcanzar a partir de la reeducación, que podrá hacer transformación; y no se podrá hacer ese cambio por la razón o el poder; son otros tiempos, contextos diferentes. Concluye que los cambios organizacionales tienen que girar alrededor del individuo y de los valores que pueden moverlo hacia la nueva cultura, basado en la delegación de autoridad, comunicación abierta, participación, colaboración y aprendizaje continuo. Según los resultados, podemos precisar que los servidores públicos administrativos de las Unidades de Gestión Educativa Local, presentan seria resistencia, el diagnóstico organizacional, pudo identificar que los valores mejora, mayor responsabilidad, actitud de competencia, de mayor compromiso, puntualidad; no están sentadas para comenzar el camino del cambio organizacional, toda vez que la conformidad, confort, aversión al riesgo son fuertes y presentes, sumado que la edad no es determinante para generar un cambio; por el contrario, tiene que, darse un paso previo, iniciar un proceso de alineación integral de esos valores a fin de modificar la cultura y hacerla proclive al cambio, disminuyendo la resistencia e incrementando las posibilidades de éxito.

Según López, Restrepo de Ocampo \& López Velásquez (2013) la resistencia al cambio en organizaciones modernas, solo se podrá lograr el proceso de cambio; si se inicia con el cambio personal y al interior, además de como las organizaciones modernas son conscientes de ello. El cambio, es un proceso a través del cual se pasa de un estado a otro, generándose modificaciones o alteraciones de carácter cuantitativo y/o cualitativo de la realidad. El cambio, es un fenómeno social que ocurre en diversos contextos, con diferentes magnitudes, y con variados tipos y manifestaciones. Los servidores públicos administrativos de las Unidades de Gestión Educativa Local, dados los resultados presentan resistencia, conformidad, aversión al riesgo por competir, mejorar, sumado que la edad temprana de un servidor público administrativo no es determinante para incidir en el cambio personal, además se tiene una cultura de confort, ceñido a sus comodidades, rutina. La resistencia al cambio en los servidores públicos administrativos de las Unidades de Gestión Educativa Local, en algunas ocasiones se muestra abierto, se manifiesta en huelgas, estandarizado rendimiento (menor productividad) o se muestra encubierta a través de comportamientos como demoras, presión por ausentarse, solicitudes de traslado, pérdida de la motivación, moral más baja y tasas considerables de errores. En este sentido se expresa que una de las formas más dañinas de la resistencia es el limitado compromiso, porque solo se sujetan a cumplir lo estipulado en la norma, directivas, sin agregar un valor agregado o procedimiento que permita facilitar una práctica o función administrativa.

\section{CONCLUSIONES}

A nivel de la región Apurímac, en las Unidades de Gestión Educativa Local, no existen diferencias significativas entre ellas, respecto a la predisposición al cambio organizacional de parte de los servidores públicos administrativos, todas presentan un mismo comportamiento de conformidad, confort, aversión al riesgo, si bien existe una estructura flexible 
de comunicación e informalidad, ello no es favorable directamente para iniciar un cambio, además los comportamientos abiertos y encubiertos son notorios, dando una objetividad a la resistencia al cambio organizacional. Todos los valores p-valor obtenidos son superiores a 0.05 , lo cual indica que las medias son iguales o muy próximas de ambas muestras de UGEL comparadas. Por lo tanto, siempre existirá una débil predisposición por lograr un cambio organizacional, porque la rutina ligada al conformismo, fortalecen el confort, la aversión al riesgo, por el miedo a competir; son fuertes y dominan el comportamiento de los servidores públicos administrativos ubicados en las Unidades de Gestión Educativa Local. Además, se confirma que el tiempo de servicio del trabajador (mayor edad) no es determinante que presente una mayor resistencia al cambio.

\section{REFERENCIAS}

Acosta, C. A. (2002). Cuatro preguntas para iniciarse en cambio organizacional. Revista colombiana de psicologia, (11), 9-24. https://www.redalyc.org/pdf/804/80401101. pdf

Barba, A. (2000). Cambio organizacional y cambio en los paradigmas de la administración. Iztapalapa: Revista de Ciencias Sociales y Humanidades, (48), 1134. https://dialnet.unirioja.es/descarga/articulo/7062858.pdf

Blanchard, K., \& O'Connor, M. (1997). Administración por Valores. Editorial Norma.

Carrasco Díaz, S. (2005). Metodología de la Investigación Cientifica (pautas metodológicas para diseñar y elaborar el proyecto de investigación). UNMSM.

Daft, R. (1998). Organizational Theory and Design. (6 ${ }^{a}$. Edición). Southwestern.

Díaz-Barrios, J. (2005). Cambio organizacional: Una aproximación por valores. Revista venezolana de gerencia, 10(32), 605-627. https://www.redalyc.org/ pdf/290/29003205.pdf

Gibson, I. J., \& Donnelly, J. (1996). Las organizaciones, comportamiento, estructura, procesos ( $8^{\mathrm{a}}$. Edición). Mc Graw Hill.

López, M. E., Restrepo de Ocampo, L. E., \& López Velásquez, G. L. (2013). Resistencia al cambio en organizaciones modernas. Scientia et technica, 18(1), 149-157. http:// revistas.utp.edu.co/index.php/revistaciencia/article/viewFile/7159/5307

Hernández, S. R, Fernández, C. C. \& Baptista, L.P. (2014). Metodología de la investigación (sexta edición). Mc Graw Hill.

Jones, G. R., Ruiz Diaz, C., Solares, F., \& Spencer, E. (2008). Teoría organizacional: diseño y cambio en las organizaciones. Pearson Educación.

Jones, Gareth (2008). Teoría organizacional: diseño y cambio en las organizaciones. Pearson education. Pretince hall. https://luisvaldiviesomerino.files.wordpress. com/2016/08/teoria-organizacional-5ta-ed-jones-1.pdf.

Ortiz F. G., \& García M. (2008). Metodología de la investigación. LIMUSA. 